\title{
Competitividad en costos: postensado en losas
}

\author{
Hurtado J. ${ }^{1}$; Morales S. ${ }^{2}$; Hernández L. ${ }^{3}$ \\ ${ }^{1}$ Ingeniero Civil, Escuela Politécnica Nacional, Facultad de Ingeniería Civil y Ambiental, Quito, Ecuador \\ e-mail: jaha@outlook.es \\ ${ }^{2}$ Ingeniero Civil, Escuela Politécnica Nacional, Master of Science in Structural Engineering, \\ Universidad de Florida, Florida, USA. \\ e-mail: sebastianmorales@grupodelpacifico.com.ec \\ ${ }^{3}$ Profesor titular, Escuela Politécnica Nacional, Facultad de Ingeniería Civil y Ambiental, Quito, Ecuador. \\ e-mail: luis.hernandezr@epn.edu.ec
}

\begin{abstract}
Resumen
El presente estudio técnico compara dos modelos de edificios con las mismas características arquitectónicas para evaluar las ventajas económicas que se obtienen al emplear un sistema de losas de hormigón con acero postensado con respecto a las de hormigón armado tradicional, bajo todas las provisiones y requisitos que se establecen en los distintos códigos y normas de la construcción (ACI 318-14, NEC 2015 y PTI). El edificio que se va a comparar utiliza un sistema dual de pórticos especiales resistentes a momentos y muros especiales de corte que formarán conjuntamente el sistema resistente a fuerzas laterales.

También se realizó una comparación económica para losas aisladas de hormigón armado además de la planta tipo con diferentes luces mostrando gráficamente mediante curvas y barras el ahorro en cantidades de materiales que se puede alcanzar mediante el uso del sistema postensado, mostrándose así, como una alternativa eficiente y económica para su aplicación en la industria de la construcción.
\end{abstract}

Palabras clave: hormigón postensado, sistema dual, hormigón presforzado, comparación económica.

\begin{abstract}
A comparison between two models of buildings with the same architectural characteristics is presented to evaluate the economic advantages which are obtained by using a system of postensioned slabs, under all provisions and requirements that are established in the different codes and construction standards (ACI 318-14, NEC 2015 \& PTI). The building to be compared is composed as a dual system of special moment frame in combination with special structural walls that together conforms the lateral force resistant system.

Also, the economic comparison was made for insulated reinforced concrete slabs and a typical plant with different spans showing graphically through curves and bars the savings in material quantities that can be achieved through the use of the post-tensioned system, thus showing an efficient and economical alternative for its application in the construction industry.
\end{abstract}

Keywords: postensioned slabs, dual system, prestressed concrete, economic comparison. 


\section{Introducción}

En la actualidad el desarrollo de los materiales utilizados en la construcción a un costo relativamente bajo ha permitido obtener acero de mayor resistencia al convencional y de la misma manera se ha logrado producir hormigones con mayor resistencia a la compresión. La combinación del hormigón en conjunto con torones de presfuerzo de alta resistencia colocados bajo una trayectoria predefinida y tensados una vez que el hormigón adquiere una resistencia mínima, permite introducir esfuerzos en el elemento que contrarrestan los efectos producidos por cargas gravitacionales, mejorando así la capacidad resistente del elemento [1]. El acero es un material que trabaja bajo tensiones altas después del tensado. El concreto es un material frágil a tensión cuya capacidad se ve mejorada con la inclusión del acero sin que su capacidad a compresión se vea afectada [2].

El desarrollo de estos materiales permite una tendencia a progresar hacia estructuras más económicas mediante métodos de diseño mejorados y también con el uso de materiales de alta resistencia. Los resultados permiten la reducción de las secciones y por ende la reducción del peso, donde esta representa una parte sustancial de la carga total [3].

En vista de la necesidad que tiene el Ecuador de implementar nuevos sistemas constructivos que impulsen a la industria de la construcción incorporando a sus procesos ventajas en cuanto a economía, seguridad y eficiencia se refiere, la inclusión de acero postensado en el sistema de entrepiso resulta una alternativa viable que ha sido utilizada ampliamente en países de Latinoamérica como: Brasil, Chile, Colombia, Panamá y Perú.

\section{Bases técnicas de comportamiento y comparación}

Tomando en cuenta la necesidad de hacer una comparación económica del sistema postensado con respecto al sistema de hormigón armado tradicional, se ha realizado un estudio donde para varias luces se ha diseñado lozas macizas de hormigón armado y losas postensadas sobre vigas. Adicionalmente, se compara una edificación de diez pisos para uso residencial y diseñado cum- pliendo todos los parámetros y requisitos estipulados en la Norma Ecuatoriana de la Construcción (NEC 2015) y parámetros para postensado estipulados en por el Post-Tensioning Institute (PTI, 2006).

La concepción de un adecuado sistema resistente de fuerzas laterales es fundamental para el análisis y comportamiento de un modelo o edificio. La experiencia obtenida con los eventos naturales suscitados en el país ha determinado que es necesario un sistema que rigidice la estructura y controle de manera eficiente las derivas de piso para una zona de alta amenaza sísmica.

Se presenta un sistema dual que combina pórticos especiales a momento y muros especiales a cortante los cuales resistirán las cargas laterales del sismo de diseño en proporción a sus rigideces, los pórticos especiales a momento deben ser capaces de resistir por lo menos un $25 \%$ de las fuerzas de sismo de diseño [4].

Para este caso específico, la participación de materiales de alta resistencia como el postensado no forma parte de estos sistemas resistentes debido a que son menos dúctiles. Sin embargo, se acepta su uso en el sistema resistente de fuerzas laterales siempre y cuando no exceda una contribución de $25 \%$ en resistencia de acero de presfuerzo, el resto debe resistirse con acero convencional [4].

El uso de losas con acero postensado es permitido debido a que estos elementos estructurales trabajan únicamente bajo cargas gravitacionales. Es así como se ha llegado a formar incluso edificios donde el $100 \%$ de resistencia para el sismo de diseño lo absorben los muros de corte dejando que las losas formen únicamente el sistema de gravedad.

Las ventajas que ofrece el sistema postensado en sistemas de gravedad como lo las losas son:

- $\quad$ Para las losas, la sustitución a un sistema postensado permite reducir hasta en un tercio el espesor de la sección. 
- La cantidad de acero de refuerzo se reduce entre un $60-85 \%$.

- Ciclos de construcción más rápidos [5].

Además, la aplicación del postensado en la construcción de estructuras no plantea mayores dificultades en la construcción de un edificio comparándolo con otro método constructivo [6]. Y para edificios con gran cantidad de pisos la reducción en costos puede ser substancial ya que se tendrán menores cargas en cimentaciones y menores cargas sísmicas debido a la reducción de peso que representa el uso de este sistema [7].

El diseño de losas consta de varios métodos, uno de los métodos adoptados es el Diseño por bandas o "Strip Method Design". Este método se ha optado como óptimo para el diseño de losas en hormigón armado y losas con acero postensado por la consideración e integración que tiene este método de los momentos que se ejercen en la losa. A continuación se detallará este método con sus consideraciones.

\subsection{Método de diseño por bandas - Strip Method Design}

Strip Method Design es el método adaptado para el análisis y diseño de las losas tanto de hormigón armado como losas postensadas.

El Strip Method Design es un método de diseño que permite considerar a la losa como si estuviera compuesta por un sistema de fajas en dos direcciones en ángulo recto, lo que permite calcular los momentos flexionantes por métodos simples de estática que implican el equilibrio de fajas.

La ecuación de equilibrio para una losa corresponde a:

$$
\frac{\delta^{2} m x}{\delta x^{2}}+2 \frac{\delta^{2} m x y}{\delta x \delta y}+\frac{\delta^{2} m y}{\delta y^{2}}=-w
$$

Donde $\mathrm{x}$ e y son los ejes rectangulares al plano de la losa, $m x$ y my son los momentos flexionantes por unidad de ancho en las direcciones $\mathrm{x}$ e $\mathrm{y}$, es $m x y$ el momento de torsión por unidad de ancho en las direcciones $\mathrm{x}$ e $\mathrm{y}, \mathrm{y} w$ es la carga uniformemente distribuida por área unitaria. La forma más común de obtener estos momentos en losa es mediante el análisis de elementos finitos.

El Strip Method Design tiene dos características. La primera es la facilidad con que se pueden obtener los momentos en la losa y las cargas sobre el sistema de soporte utilizando la estática simple. La segunda es la variedad de distribuciones de momentos y cargas, dependiendo posiblemente de la manera como se supone que actúa la dispersión de la carga. Cada distribución debe ser tratada según su naturaleza y cada diseñador debe suponer el comportamiento de la misma en base a la teoría elástica de distribución de momentos para asegurarse de una razonable distribución de momentos resultantes.

El uso de Strip Method Design proporciona una solución exacta que, además, utiliza eficientemente y económicamente el refuerzo [8].

La losa diseñada bajo este método va a ser reforzada por acero dúctil en las direcciones $\mathrm{x}$ e y obtienen capacidad para soportar los momentos $M_{r y} y M_{r x}$ por unidad de ancho tributario [9]. Estos momentos corresponden a:

$$
\begin{aligned}
& M_{r y}=m y \pm|m x y| \\
& M_{r x}=m x \pm|m x y|
\end{aligned}
$$

Los momentos $M_{r y}$ y $M_{r x}$ son integrados en todo el ancho tributario por la "strip" en toda su longitud obteniéndose así los momentos de diseño de la losa. Lo cual facilita los procesos de cálculo donde se considere la participación del momento torsionante en estas losas. El acero de refuerzo que se colocará en la losa es proporcional al momento de diseño por ancho tributario.

El refuerzo obtenido se distribuye ortogonalmente a lo largo de las "strips" de modo que el refuerzo total provisto en una banda es suficiente para resistir el momento factorizado total calculado para esa banda, y el momento en que la resistencia por ancho de unidad en la banda es al menos dos tercios del momento máximo por ancho de unidad en la banda, como se calcula en el análisis de elementos finitos. 


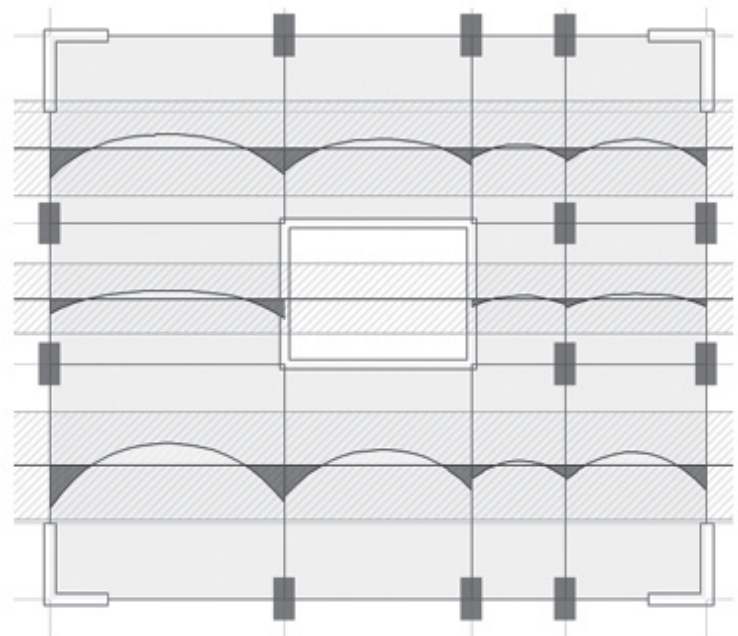

Figura 1. Strip Method Design - Strip X.

Para ilustrar estas ventajas económicas que se insertan con el sistema de losas postensadas no adherentes se hizo un análisis comparativo de los costos directos de construcción (excluyendo los costos administrativos, imprevistos y de utilidad) de losas macizas de hormigón armado o postensadas sobre vigas con varias luces y de un edificio de 10 pisos diseñado bajo las consideraciones necesarias de sismo resistencia para hormigón armado tradicional con losas macizas de hormigón y un segundo diseño con losas postensadas macizas.

\subsection{Consideraciones de diseño para losas macizas sobre vigas}

Para el diseño de losas macizas sobre vigas diseñadas a partir del método anteriormente mencionado y una vez cumplidos todos los requerimientos de serviciabilidad y resistencia se ha logrado determinar las cantidades de materiales de hormigón y acero de refuerzo que estas requieren. Los precios directos que se utilizan para esta comparación son los que se mencionan en la revista de la Cámara de la Industria de la Construcción (CAMICON).

Para la comparación de estas losas macizas se han tomado las siguientes combinaciones de luces de acuerdo a la tradición constructiva en Ecuador y además de losas con luces más grandes que no son comunes en el medio.

\begin{tabular}{|c|c|}
\hline Area & Luces \\
\hline $\mathrm{m}^{2}$ & $\mathrm{~m}$ \\
\hline 16.00 & $4 \times 4$ \\
\hline 32.00 & $8 \times 4$ \\
\hline 40.00 & $10 \times 4$ \\
\hline 48.00 & $8 \times 6$ \\
\hline 64.00 & $8 \times 8$ \\
\hline 80.00 & $10 \times 8$ \\
\hline
\end{tabular}

Figura 2. Combinaciones de luces consideradas.

En base al diseño de las losas, con estas luces se ha logrado obtener la siguiente curva de tendencia de costos directos en cuanto a costos directos con respecto a sus luces.

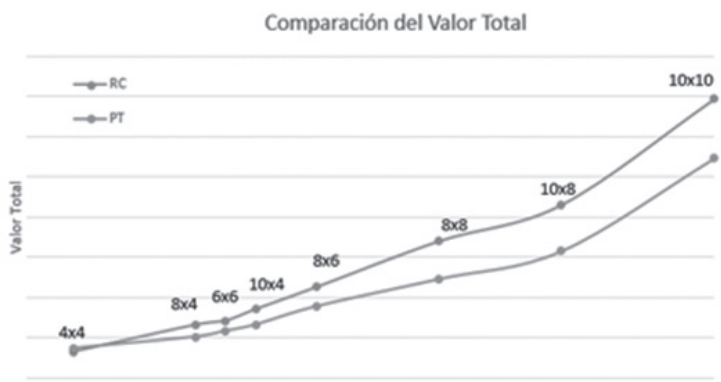

Figura 3. Curva de tendencia de costos directos.

En la curva se observa que el costo directo que se obtiene con el sistema postensado es menor. Además, el beneficio que se alcanza con el cambio a postensado es mayor en cuanto más grandes son las luces que se adaptan al proyecto arquitectónico. Por otro lado, para luces pequeñas el cambio a postensado no representa un beneficio económico en cuanto a cantidades de materiales refiere. La siguiente figura muestra mediante barras el ahorro que se genera con el cambio a postensado 
evidenciando que para luces pequeñas no existe un beneficio.

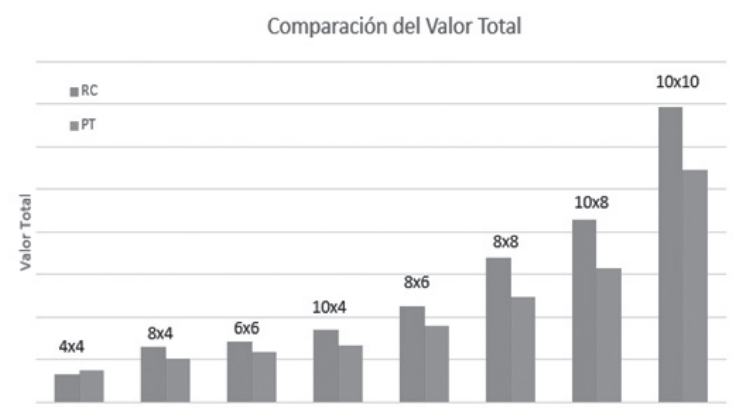

Figura 4. Diferencia de costos directos.

La gráfica anterior muestra que, de los análisis realizados se ha determinado que el porcentaje de ahorro para losas macizas sobre vigas de hormigón varían entre un 10-20\%, esta ventaja económica en conjunto con la combinación de luces más grandes convierte a ese sistema en una solución eficiente en cuanto a costos y arquitectura.

2.3 Comparación de losas macizas sobre vigas en un edificio de 10 pisos

El modelo que se plantea para la comparación económica utiliza un sistema dual con muros de corte en combinación con pórticos especiales de momento. Este sistema es implementado en zonas de alta amenaza sísmica debido a la rigidez y control de derivas que los muros de corte aportan a la estructura.

Un sistema Dual es una combinación de muros estructurales y pórticos resistentes a momentos, los cuales resistirán las cargas de sismo de diseño en proporción a sus rigideces, los pórticos especiales a momento deben ser capaces de resistir por lo menos un $25 \%$ de las fuerzas de sismo de diseño [4].

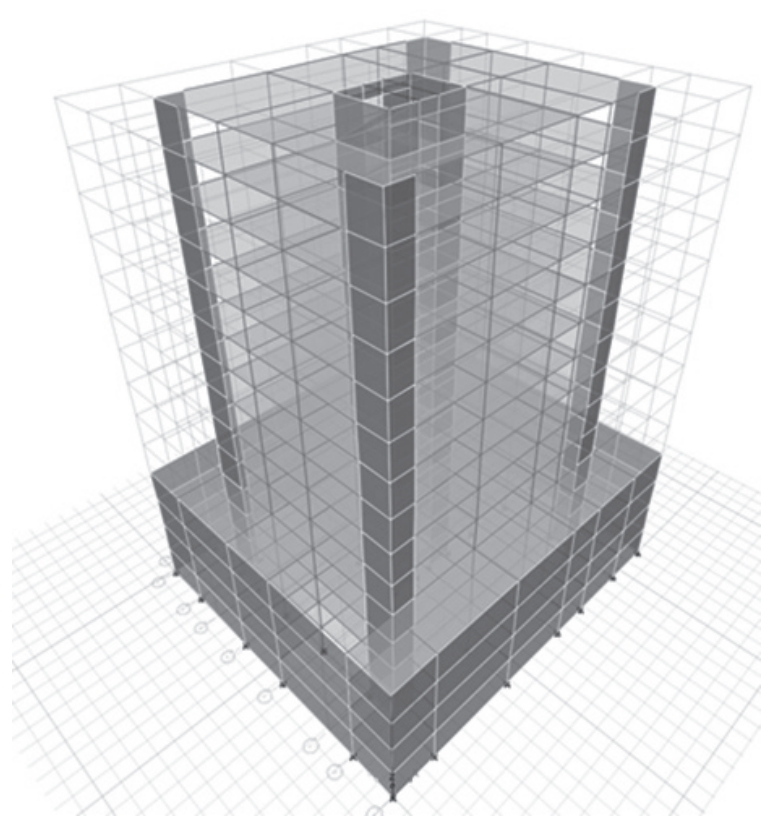

Figura 5. Modelo estructural para sistema de entrepiso macizo sobre vigas.

Esta definición es totalmente contraria a la que se dicta en el NEC - 2015 que dice: "Sistema resistente de una estructura compuesta tanto por pórticos especiales sismo resistentes como por muros estructurales adecuadamente dispuestos espacialmente, diseñados todos ellos para resistir las fuerzas sísmicas. Se entiende como una adecuada disposición ubicar los muros estructurales lo más simétricamente posible, hacia la periferia y que mantienen su longitud en planta en todo lo alto de la estructura. Para que la estructura se considere como un sistema dual se requiere que los muros absorban al menos el 75\% del corte basal en cada dirección" [11].

Esta discrepancia en cuanto a las definiciones además de los valores para los factores de reducción " $\mathrm{R}$ " que es asignado para este tipo de estructuras. Pueden provocar errores en cuanto a la ductilidad que estos modelos alcanzan. Un sistema dual no puede tener un factor " $\mathrm{R}$ " de 8 como se especifica puesto que los muros de corte son muy rígidos y no presentan ductilidad, y es precisamente lo que NEC - 2015 sugiere. Una estructura con una proporción de muros mayor al 75\% se vuelve 
menos dúctil, por ende, tendrá un factor de reducción menor a 8. La ASCE recomienda para este tipo de sistemas un factor de reducción " $\mathrm{R}$ " de 7 para pórticos especiales a momentos y de 6 para pórticos ordinarios. Es importante recalcar que la norma ecuatoriana no especifica este tipo de pórticos.

En un sistema dual la adición de columnas otorga ductilidad a los muros de corte y es lo permite aumentar su factor " $R$ ", la recomendación para sistemas de muros de corte es de "R" de 5 [10].

El modelo planteado como se observa en la figura 4, se analiza bajo las consideraciones necesarias para hormigón armado tradicional con losas macizas de hormigón y posteriormente mediante un segundo análisis con losas postensadas macizas. La implementación de un adecuado sistema de fuerzas laterales permite la inclusión de herramientas que ofrezcan un beneficio económico, así como lo es la inclusión de acero postensado. La alta amenaza sísmica y la experiencia han demostrado que el sistema de pórticos tiene un desempeño deficiente en cuanto a control de derivas en este tipo de zonas, por lo que un adecuado sistema de fuerzas resistentes permitirá satisfacer los parámetros de seguridad que se busca con el diseño sismo resistente.

La reducción de peso del edificio tiene una incidencia directa en la reducción de las fuerzas laterales que el edificio va a soportar lo cual en una zona de alta amenaza sísmica se convierte en una ventaja estructural.

\section{Resultados y discusión}

\subsection{Comportamiento estructural}

El comportamiento obtenido del análisis de ambas estructuras ha permitido determinar que la inclusión de muros y el cambio de sistema de entrepiso (reducción del peso del edificio) han resultado en un mejor control de derivas, modos de vibración y torsión en planta. Estas ventajas estructurales ofrecen un mejor resultado final y un adecuado comportamiento bajo solicitaciones sísmicas.
En cuanto a la participación de pórticos especiales para el sistema dual se ha alcanzado el siguiente porcentaje de participación:

Tabla 1. Porcentaje de participación de sistema dual - Modelo hormigón armado

\begin{tabular}{|c|c|c|c|c|}
\hline Sentido & $\mathrm{Vb}$ & Pórtico & Muro & $\%$ \\
\hline $\mathrm{X}$ & 2163.78 & 541.51 & 1622.27 & $25.03 \%$ \\
\hline $\mathrm{Y}$ & 2163.78 & 752.53 & 1411.25 & $34.78 \%$ \\
\hline
\end{tabular}

Tabla 2. Porcentaje de participación de sistema dual - Modelo hormigón postensado

\begin{tabular}{|c|c|c|c|c|}
\hline Sentido & Vb & Pórtico & Muro & $\%$ \\
\hline$X$ & 1913.75 & 478.98 & 1434.77 & $25.03 \%$ \\
\hline$Y$ & 1913.75 & 590.57 & 1323.18 & $30.86 \%$ \\
\hline
\end{tabular}

Como se puede observar en las tablas la participación del pórtico sobre los muros cumple el $25 \%$ mínimo con lo que se puede determinar efectivamente al sistema como dual. Además, se puede observar que el cambio a postensado redujo en un $12 \%$ la solicitación de cortante basal, esto se logró al alcanzar menores secciones en los elementos de hormigón que conforman el pórtico especial a momento.

La reducción de secciones de hormigón se tradujo en una reducción de un $15 \%$ para vigas y un $14 \%$ para columnas aproximadamente.

En cuanto al control de derivas se ha obtenido las mayores derivas en el eje $\mathrm{Y}$ registrándose los siguientes porcentajes para los dos modelos analizados:

\begin{tabular}{|c|c|c|c|r}
\hline Nivel & Carga & Dirección & Deriva & $\begin{array}{r}\text { Derivi } \\
\text { Inelasti }\end{array}$ \\
\hline P10 & SPECTRO Y Max & Y & 0.00224 & $1.57 \%$ \\
\hline P9 & SPECTRO Y Max & Y & 0.002376 & $1.66 \%$ \\
\hline P8 & SPECTRO Y Max & Y & 0.002514 & $1.76 \%$ \\
\hline P7 & SPECTRO Y Max & Y & 0.00262 & $1.83 \%$ \\
\hline P6 & SPECTRO Y Max & Y & 0.002685 & $1.88 \%$ \\
\hline P5 & SPECTRO Y Max & Y & 0.002701 & $1.89 \%$ \\
\hline P4 & SPECTRO Y Max & Y & 0.002655 & $1.86 \%$ \\
\hline P3 & SPECTRO Y Max & Y & 0.00254 & $1.78 \%$ \\
\hline P2 & SPECTRO Y Max & Y & 0.002326 & $1.63 \%$ \\
\hline P1 & SPECTRO Y Max & Y & 0.001904 & $1.33 \%$ \\
\hline & & & & \\
\hline
\end{tabular}

Tabla 3. Derivas - Modelo hormigón armado 
Tabla 4. Derivas - Modelo hormigón postensado

\begin{tabular}{|c|c|c|c|c|}
\hline Nivel & Carga & Dirección & Deriva & $\begin{array}{c}\text { Deriva } \\
\text { Inelastica }\end{array}$ \\
\hline P10 & SPECTRO Y Max & $Y$ & 0.002275 & $1.59 \%$ \\
\hline P9 & SPECTRO Y Max & $Y$ & 0.002383 & $1.67 \%$ \\
\hline P8 & SPECTRO Y Max & $Y$ & 0.002486 & $1.74 \%$ \\
\hline P7 & SPECTRO Y Max & $Y$ & 0.00256 & $1.79 \%$ \\
\hline P6 & SPECTRO Y Max & $Y$ & 0.002599 & $1.82 \%$ \\
\hline P5 & SPECTRO Y Max & $Y$ & 0.002591 & $1.81 \%$ \\
\hline P4 & SPECTRO Y Max & $Y$ & 0.002529 & $1.77 \%$ \\
\hline P3 & SPECTRO Y Max & $Y$ & 0.002407 & $1.68 \%$ \\
\hline P2 & SPECTRO Y Max & $Y$ & 0.002196 & $1.54 \%$ \\
\hline P1 & SPECTRO Y Max & $Y$ & 0.0018 & $1.26 \%$ \\
\hline 0 & SPECTRO Y Max & $Y$ & 0.000259 & $0.18 \%$ \\
\hline
\end{tabular}

Con la reducción de secciones en el modelo considerado se ha logrado controlar las derivas de piso según los límites establecidos en la norma ecuatoriana de la construcción (NEC, 2015). Evidentemente este control tiene que ver directamente con la reducción de fuerzas laterales a la estructura alcanzada con el cambio a postensado.

Respecto a los modos de vibración, se ha logrado una pequeña participación en cuanto a los modos de vibración con el cambio a entrepiso postensado, como se observa a continuación:

Tabla 5. Modos de vibración - Modelo hormigón armado

\begin{tabular}{|c|c|c|c|c|}
\hline Modal & Periodo & Ux & Uy & $\mathrm{Rz}$ \\
\hline 1 & 1.181 & 0 & 0.996 & 0.004 \\
\hline 2 & 1.053 & 0.995 & 0 & 0.005 \\
\hline 3 & 0.814 & 0.005 & 0.004 & 0.991 \\
\hline
\end{tabular}

Tabla 6. Modos de vibración - Modelo hormigón postensado

\begin{tabular}{|c|c|c|c|c|}
\hline Modal & Periodo & Ux & Uy & Rz \\
\hline 1 & 1.18 & 0 & 0.998 & 0.002 \\
\hline 2 & 1.048 & 0.997 & 0 & 0.003 \\
\hline 3 & 0.774 & 0.003 & 0.002 & 0.996 \\
\hline
\end{tabular}

Alcanzado un comportamiento estructural adecuado y una vez cumplidas con las provisiones para pórticos especiales a momentos referidas en los capítulos 18 y 25 del Código American Concrete Institute (ACI 318-14, 2014) se ha logrado determinar las cantidades de materiales que estas losas requieren y comparar estas cantidades tanto para el modelo de entrepiso macizo de hormigón armado y de hormigón postensado sobre vigas.

\subsection{Competitividad en costos}

De las ventajas anteriormente mencionadas, respecto a la reducción de secciones y cuantías de acero obtenidas para ambos modelos analizados, una vez cumplidos todos los requerimientos de la estructura en cuanto a efectos de sismo resistencia refiere, se ha podido determinar la siguiente gráfica:

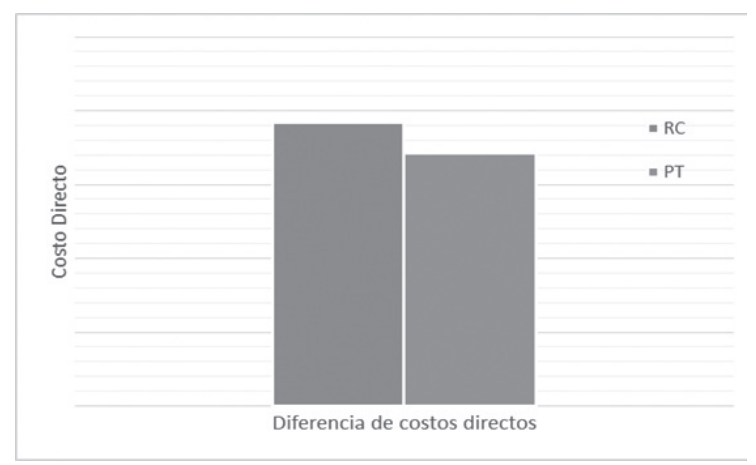

Figura 6. Comparación económica en sistema de entrepiso

Como se puede observar en la figura, mediante el empleo de un sistema de losas postensadas macizas para un sistema dual se ha podido alcanzar un ahorro aproximado de un 10\% del valor total.

El cambio de entrepiso postensado permitió obtener secciones de menor tamaño y menores cuantías de acero lo que se traduce en una reducción de peso y, por ende, una menor carga sísmica para el edificio, todo esto en conjunto permitió una ventaja económica en la estructura.

Este ahorro representativo en comparación al hormigón armado tradicional convierte al sistema postensado en una alternativa económica viable para los procesos constructivos del país en un sistema dual adecuado para zonas de alta amenaza sísmica. Estas ventajas mencionadas no consideran la rapidez constructiva que varios autores refieren y aumentan el porcentaje de ahorro alcanzado con el cambio a postensado.

El ahorro se incrementa si se toman en cuenta los beneficios de rapidez constructiva, los mismos que reducen los tiempos de construcción ya que el postensado permite un desencofrado acelerado de los entrepisos y, además, reducen la mano de obra. 
El trabajo conjunto entre ingenieros y arquitectos es lo que se necesita para poder explotar los beneficios económicos que este sistema puede ofrecer; el uso de herramientas tecnológicas que permitan este trabajo conjunto son necesarias para avanzar en los procesos constructivos.

La tradición constructiva en el país es el empleo de losas alivianadas las mismas que reducen en gran proporción la cantidad de hormigón empleada en contraste con las losas macizas. El sistema de losas postensadas puede adaptarse a este método constructivo y brindar los beneficios económicos anteriormente mencionados siempre $y$ cuando se asegure su correcto procedimiento de cálculo y diseño.

\section{Conclusiones y recomendaciones}

Un correcto entendimiento de los sistemas resistentes de fuerzas laterales es necesario en zonas de alta amenaza sísmica. Una correcta definición de los sistemas resistentes y un adecuado factor de reducción permitirán asegurar diseños eficientes y seguros acordes al comportamiento esperado de la estructura planteada.

El uso de un sistema de hormigón postensado permite reducir secciones de hormigón $y$, por ende, reducir el peso propio de la estructura, lo cual es una ventaja estructural en zonas de alto riesgo sísmico, puesto que la fuerza sísmica que soporta la estructura es directamente proporcional al peso de esta.

Las losas postensadas requieren de un eficiente sistema resistente de fuerzas laterales puesto que estas no forman parte de este. Estas losas son eficientes bajo cargas de servicio y son poco dúctiles debido a los materiales de alta resistencia que emplean.

Las ventajas económicas para un sistema que emplea losas de hormigón postensado dependen y varían desde la concepción arquitectónica, así como se observa en la figura 3 , para luces pequeñas $(4 \mathrm{~m}-6 \mathrm{~m})$ el sistema postensado se iguala económicamente al de hormigón armado tradicional, sin embargo, al modificar la arquitectura implementando luces más grandes la ventaja eco- nómica que se puede alcanzar es mayor.

La reducción de peso en la estructura genera menores fuerzas sísmicas en la misma con lo que se producen menores solicitaciones a los elementos estructurales en cuanto a momentos y cargas axiales. La disminución de solicitaciones permite secciones de elementos estructurales más pequeñas y el uso de hormigones de alta resistencia secciones más eficientes. Juntamente con la reducción de solicitaciones en los elementos también se obtienen menores cuantías de acero. Esta es la ventaja económica que el cambio a losas de hormigón postensadas permite alcanzar.

El empleo de losas postensadas planas con un sistema de muros de corte ha dado resultados adecuados en otros países de alto riesgo sísmico en donde el uso de este sistema es de vieja aplicación y ha dado resultados satisfactorios además de ventajas económicas y arquitectónicas para su desarrollo.

El país de cara al desarrollo urbano debería adaptar el sistema postensado en cuanto a los beneficios económicos que este presenta y la arquitectura que permite alcanzar. Es necesario cambiar los paradigmas constructivos y empezar a crear soluciones alternativas eficientes y seguras basadas en la experiencia y ventajas que otros países han obtenido de este sistema.

\section{Referencias}

[1] Aalami, B. (2014). Post-Tensioned Buildings, Design and Construction (International ed.). USA: PT-Structures.

[2] Lin T.Y, B. N. (2010). Design of Prestressed Concrete Structures (3rd ed.). USA: Wiley India.

[3] Nilson Arthur, D. D. (2010). Design of Concrete Structures (14th ed.). New York: McGraw Hill.

[4] Moehle, J. (2014). Seismic Desing of Reinforced Concrete Buildings. USA: Mc Graw Hill.

[5] Nawy, E. (2009). Prestressed Concrete (5th ed.). New Jersey, Estados Unidos: Pearson. 
[6] FIB. (2005). Post - tensioning in Buildings. Stuttgart: Sprint - Digital - Druck.

[7] PTI. (2006). Post-Tensioning Manual. Phoenix: PTI.

[8] Gamble, P. (2000). Losas de concreto reforzado. USA.
[9] Park R, G. W. (1990). Losas de concreto reforzado. USA.

[10] ASCE, 7.-1. (2017). Minimum Design Loads and Associated Criteria for Buildings and Other Structures. USA.

[11] NEC. (2015). Diseño sismo resistente. Norma Ecuatoriana de la Construcción, 139. 